\title{
Enhanced Sorting on Fully Homomorphic Encrypted Data
}

\author{
Rashmi Salavi and Dr.M.M. Math
}

\begin{abstract}
The challenge of maintaining confidentiality of stored data in cloud is of vital importance to realize the potential of cloud computing. Storing data in encrypted form may solve the problem, but increases the security issues. Performing operations on cloud data requires repeated decryption-encryption which lessens the meaning of cloud. Hence, Fully homomorphic encryption (FHE) is an effective scheme to support arbitrary operations directly on encrypted data. It is propose the implementation of various algorithms that sort data encrypted with Fully Homomorphic Encryption scheme. The technique is obtained by focusing on the multiplicative depth of the sorting circuit along with the more traditional metrics such as number of comparisons and number of iterations. The reduced depth allows significant reduction in the growth of noise resulting in approximately $50 \%$ efficiency savings. It is first to consider a number of well known comparison based sorting algorithms as well as some sorting networks, and analyze their circuit implementations with respect to multiplicative depth.
\end{abstract}

Keywords--- Cloud, Fully Homomorphic Encryption (FHE), Hcrypt.

\section{INTRODUCTION}

$\mathrm{C}$ RYPTOGRAPHIC applications to secure data stored and managed on the cloud environment have been gaining significant importance after Gentry's invention of Fully Homomorphic Encryption in 2009 [1]. FHE algorithms allow performing arbitrary computations on the encrypted data and still preserving the confidentiality of the data.

Still research on efficient FHE algorithms and their analysis are at work [2][3] [14][15][16][17]. However, the practicality of FHE is not in progress, which is the prime motivation for our research work.

Study of applications of FHE has been an area of pursuit in the last few years mainly because of its impact on cloud computing security [4], [5], [6], [7]. To our knowledge, the idea of sorting an array of encrypted integers was first discussed by Carlos et al. [8]. Here authors explicitly presented control flow of Bubble sort algorithm that can sort encrypted data. Later Chatterjee et al. [9] used Insertion and Bubble sorting techniques to sort an array of encrypted integers of size 5-40 by using herypt [10] library. This library was programmed in C and is based on FHE scheme proposed by Smart and Verteceuran [11], which has relatively smaller

Rashmi Salavi, Research Scholar, Dept. of Computer Science \& Engg., KLS Gogte Institute of Technology, Udyambag, Belagavi.

Dr.M.M. Math, Professor, Dept. of Computer Science \& Engg., KLS Gogte Institute of Technology, Udyambag, Belagavi.

DOI:10.9756/BIJSESC.8265 key and ciphertext sizes and yet, following Gentry's bootstrapping blueprint [1] of developing a FHE scheme from a Somewhat Homomorphic Encryption scheme. Chatterjee et al. [9] also proposed a sorting technique called LazySort which claims a substantial performance improvement compared to the classical Bubble and Insertion sorting techniques. This paper focuses on how far one can reach on sorting data in the encrypted domain with the sorting algorithms used to sort plaintexts.

\section{BRIEF OVERVIEW OF A FHE SCHEME}

A public key FHE scheme $\xi: \mathrm{M} \rightarrow \mathrm{C}$ is described by a tuple of four algorithms i.e.

\section{$\xi=$ (KeyGen, Encrypt, Decrypt, Evaluate)}

where KeyGen, Encrypt, Decrypt denote the key generation, encryption and decryption functions of $\xi$ respectively.

KeyGen: First choose sequence of primes $\mathrm{q} 0>\mathrm{q} 1>\ldots>$ qd to use a different $\mathrm{q}_{\mathrm{i}}$ in each level. A public and secret key pair is computed for each level:

$$
h^{(i)}=2 g^{(i)}\left(f^{(i)}\right)^{-1} \text { and } f^{(i)}=2 u^{(i)}+1
$$

where $\left\{g^{(i)}, f^{(i)}\right\} \in X$.X indicates an error distribution.

Encrypt: To encrypt a bit $\mathrm{b}$ for the ith level computation required is: $\mathrm{c}^{(\mathrm{i})}=\mathrm{h}^{(\mathrm{i})} \mathrm{s}+2 \mathrm{e}+b$, where $\{s, e\} \in X$.

Decrypt: In order to compute the decryption of a value for specific level $i$ is:

$$
m=c^{(i)} f^{(i)}(\bmod 2)
$$

Evaluate: The gate level logic operations XOR and AND are done by computing the addition and multiplication of the ciphertexts. In case of $c_{1}^{(i)}=\operatorname{Encrypt}\left(b_{1}\right)$ and $c_{2}^{(i)}=$ Encrypt $\left(b_{2}\right)$.

The multiplication creates a significant noise in the ciphertext and to cope with that it is need to apply Relinearization and modulus switch. Brakerski and Vaikuntanathan [4] describe a transformation - called relinearization - that reduces the ciphertext back to two ring elements. If the multiplication is performed on two ciphertexts (each containing two ring elements) produced by the encryption algorithm. The resulting ciphertext contains three ring elements. The multiplication operation turned two linear ciphertexts into a quadratic ciphertexts. The goal of relinearization is to bring this back down to a linear ciphertext. Later, the modulus switch decreases the noise bits by dividing and multiplying the new ciphertext with the previous and current moduli, respectively. 


\section{SORTING AlgORITHMS}

Sorting is one of the most important tasks in computing. Various sorting algorithms have been proposed in the literature [12]. These algorithms have been heavily investigated and characterized according to their time and space requirements, as well as to the degree of their suitability for parallelization. Most of the FHE schemes are circuit base; is is needed to add the multiplicative circuit depth metric for homomorphic sorting techniques. So there is a need to convert serial sorting algorithms into circuit by eliminating loops and conditional assignments by arithmetic's. A sorting network is a circuit which consists of comparators and swapping operations which are set in advance; means that there is no data dependency in the flow of the algorithm steps. While there are some algorithms specifically designed as a sorting network, some classical sorting algorithms can also be represented as a network, as FHE properties require. Hence it is required to go over some well known algorithms.

\section{A. Bubble Sort}

Bubble Sort is one of the simplest sorting techniques that use only primitive comparison and swap operations. Chatarjee et al. [9] design homomorphic conditional swap circuits to facilitate homomorphic evaluation of the Bubble Sort algorithm. Sorting algorithms works in passes over the array. In each pass two adjacent elements are compared and swapped to move larger element to the right. The average and worst case performance of an array of $n$ elements are $\mathrm{O}\left(\mathrm{n}^{2}\right)$. During homomorphic evaluation it is needed to make n-1 passes over the array which always leads to worst case complexity. Since the element in the rightmost portion of array is sorted, every pass decreases by one in number of comparisons and swaps. Thus, overall $[(n-1)+(n-2)+\ldots .+1]=\left(n^{2}-n\right) / 2$ comparison and swap circuit blocks are used. It is possible to overlap the passes which creates a network version of Bubble Sort, known as Odd Even Sort, by not waiting to start the next pass until a pass is finished.

\section{B. Odd-Even Sort}

In odd-even sort algorithm, there are two passes: one on even index and another on odd index. In the first pass, every even indexed element is compared and swapped with its right neighbour while in the second pass; every odd indexed element is compared and swapped with its right neighbour. Considering these two steps as a round, the identical operations are applied in each round. The total number of comparisons is $\mathrm{n}-1$ in each round, there are $\mathrm{n}$ passes which means $\mathrm{n} / 2$ rounds and so overall, there are $n(n-1) / 2$ comparators.

\section{Insertion Sort}

Insertion sort is a simple sorting algorithm that iteratively builds a sorted array from an unsorted one. The sorted array initially holds only the first element. Then each element is one by one added to the sorted list by comparing it from right to left with the elements in the sorted list until an element smaller is encountered. The new element is then inserted into the sorted array next to the first smaller element when scanning right to left. The average case and the worst case complexity of the algorithm is $\mathrm{O}\left(\mathrm{n}^{2}\right)$ while the best case is only $\mathrm{O}(\mathrm{n})$.
When considered as a circuit for homomorphic evaluation it is need to run the algorithm with the worst case complexity, without making early decisions as in Bubble Sort. The sorted array can be build by making increasing number of comparison and conditional swaps. This circuit can be used in a more efficient way by overlapping some comparisons.

\section{Merge Sort}

Merge Sort is an asymptotically faster algorithm and allows early termination in normal execution, which reduces its complexity. The algorithm is recursively applied by splitting arrays into smaller ones. In the innermost recursion, arrays of two elements are sorted, where only one comparison is needed in one subarray. In the merging step, which combines two individually sorted arrays into a single sorted array, at most three comparisons are applied in each partition. This eventually requires $\mathrm{O}(\mathrm{n} \log (\mathrm{n}))$ comparisons in the worst case. But in homomorphic evaluation, the merging step requires many more comparisons, due to lack of input knowledge. Hence need to perform $\left(n^{2}-n\right) / 2$ comparisons to sort an array of $\mathrm{n}$ elements. On the other hand, since there is no swapping, i.e. no data dependency, during the execution of a single merge step, it is possible to compute all of the comparisons in parallel at the beginning of each merge step.

\section{E. Odd-Even Merge Sort}

Odd-Even Merge Sort is a sorting network devised by Batcher [13]. It has a recursive structure similar to Merge Sort. The algorithm considers two already sorted half-lists at each merge step. In a merge step, the merging process is recursively applied to even and odd indexed elements separately while arranging them into two halves. This process continues until there is only one element in each half list in which case a comparison and swap circuit is applied in order to merge them into an array. Once the even indexed half and the odd indexed half are both internally merged, comparison and swap circuits are applied to inner adjacent elements only.

\section{F. Comparison Matrix based Sorting Algorithm}

Given the insufficiency of existing sorting algorithms, the straightforward sorting algorithm based on comparison matrix will give significant improvement in the efficiency. The algorithm take an input vector and compute the sorted vector by evaluating the sorting circuits. The circuit evaluation makes it easy to apply the SWHE algorithm for homomorphic evaluation. This circuit makes use of another circuits for equality check and comparison. This algorithm is described below:

Encrypted Input Vector: $\mathrm{E}(\mathrm{X})=\left(\mathrm{X}_{0}, \mathrm{X}_{1}, \ldots \ldots \mathrm{X}_{\mathrm{n}}\right)$

Encrypted Output Vector: $\mathrm{E}(\mathrm{Y})=\left(\mathrm{Y}_{0}, \mathrm{Y}_{1}, \ldots \ldots \mathrm{Y}_{\mathrm{n}}\right)$

Step I: Compute Comparison Matrix (M):

$$
\mathbf{M}=\left(\begin{array}{llll}
m_{0,0} & m_{0,1} & \cdots & m_{0, n-1} \\
m_{1,0} & m_{1,1} & \cdots & m_{1, n-1} \\
\vdots & \vdots & \ldots & \vdots \\
m_{n-1,0} & m_{n-1,1} & & m_{n-1, n-1}
\end{array}\right)
$$


Where $\left\{\begin{array}{lr}\left\{m_{i, i}=0 \mid\right. & \text { for all } i<n\} \\ \left\{m_{i, j}=1 \mid \text { if } X_{i}<X_{j} \text { and } i<j\right\} \\ \left\{m_{i, j}=0 \mid\right. & \text { else }\}\end{array}\right.$

The remaining entries in the lower triangular part of $\mathrm{M}$, whose indices satisfy $\mathrm{i}>\mathrm{j}$, are computed as $m_{j, i}=m_{i, j} \oplus 1$

\section{Step II: Compute Index Vector ( $\sigma$ )}

The index vector, $\sigma$, indicates the positions of the vector elements in the sorted output vector, and is computed using the comparison matrix $M$. In matrix $M$, the sum of all elements in a column gives the number of elements, with which the element with the index of the column number is greater than. For instance, the sum of all elements in column $j$ is the number of elements, with which the element $\mathrm{Xj}$ is larger than, as 1 is added to the sum for each such element. Therefore, the sum is also the index of $\mathrm{Xj}$ in the sorted output vector.

In other words, if an element is larger than $\mathrm{k}$ other elements, then this imply that it is the $\mathrm{k}+1^{\text {st }}$ largest element and its index is $\mathrm{k}$ in a zero-based output vector. Now, since all data is in an encrypted form, there is no knowledge about the elements of the $\sigma$; therefore it cannot used directly for homomorphic sorting. Here, it simply compare each element of the index vector $\sigma$, with each possible index value (which is in the interval $[0, \mathrm{n}-1])$; the equality places the corresponding input element in the current position of the output vector.

$$
Y_{j}=\sum_{i=0}^{n}\left(\sigma_{\mathrm{i}}=\mathrm{j}\right) \mathrm{X}_{\mathrm{i}} \text { for } \mathrm{j} \in \mathrm{n}
$$

Example: Let input vector $\mathrm{X}=(4,3,7,2)$.

Comparison Matrix (M) is:

The index Vector $(\sigma)$. $\left(\begin{array}{cccc}0 & 0 & 1 & 0 \\ 1 & 0 & 1 & 0 \\ 0 & 0 & 0 & 0 \\ 1 & 1 & 1 & 0\end{array}\right)$

$$
\left(\begin{array}{llll}
2 & 1 & 3 & 0
\end{array}\right)
$$

The output vector $\mathrm{Y}$ is calculated as below:

For $\mathrm{j}=2$

$$
Y_{2}=\sum_{i=0}^{n}\left(\sigma_{i}=2\right) X_{i}
$$

Therefore

$$
\begin{aligned}
& \text { For } \mathrm{j}=2 \\
& Y_{2}=(2=2) X_{0}+(1=2) X_{1}+(3=2) X_{2}+(0=2) X_{3} \\
& =(1) X_{0}+(0) X_{1}+(0) X_{2}+(0) X_{3}=X_{0}
\end{aligned}
$$$$
\text { For } \mathrm{j}=1
$$

$$
\begin{array}{r}
Y_{1}=(2=1) X_{0}+(1=1) X_{1}+(3=1) X_{2}+(0=1) X_{3} \\
=(0) X_{0}+(1) X_{1}+(0) X_{2}+(0) X_{3}=X_{1}
\end{array}
$$

For $\mathrm{j}=3$

$$
\begin{aligned}
& Y_{3}=(2=3) X_{0}+(1=3) X_{1}+(3=3) X_{2}+(0=3) X_{3} \\
& =(0) X_{0}+(0) X_{1}+(1) X_{2}+(0) X_{3}=X_{2} \\
& \text { For } \mathrm{j}=0 \\
& Y_{0}=(2=0) X_{0}+(1=0) X_{1}+(3=0) X_{2}+(0=0) X_{3} \\
& =(0) X_{0}+(0) X_{1}+(0) X_{2}+(1) X_{3}=X_{3} \\
& \text { Therefore output vector } \mathrm{Y}=\left(\begin{array}{llll}
X_{3}, & X_{1} & X_{0} & X_{2}
\end{array}\right) \\
& =\{2,3,4,7)
\end{aligned}
$$

The implementation of above depth optimized sorting method using the SWHE scheme of [11] and the performance for different number of array lengths need to measured. This paper mainly focus on the comparision of efficiency of different sorting techniques with different size of array. The depth optimized sorting techniques significantly improve the performance compared to traditional sorting algorithms.

\section{CONCLUSION}

The proposed implementation of comparision matrix based optimized sorting algorithm used for efficient homomorphic evaluation. Circuit depth is closely related to the parameter sizes in leveled homomorphic encryption implementations and therefore directly affect the overall performance of the homomorphic circuit evaluation. Existing sorting algorithms are not optimized for homomorphic evaluation. The depth analysis for several classical sorting algorithms: Bubble sort, Insertion Sort, Odd Even Sort, Odd Even Merge Sort, and Odd Even Merge Sort can be compared with optimized sorting algorithms. Due to use of relinearization and modulus switching there is significant growth in performance of optimized sorting technique.

\section{REFERENCES}

[1] C. Gentry, "A fully homomorphic encryption scheme", Ph.d. thesis, 2009.

[2] Z. Zhang, T. Plantard and W. Susilo, "Reaction attack on outsourced computing with fully homomorphic encryption schemes. In International Conference on Information Security and Cryptology, Pp. 419-436, 2011.

[3] T. Plantard, W. Susilo and H. Zhang, "Lll for ideal lattices: re-evaluation of the security of gentryhalevis fhe scheme", Vol. 76, Pp. 325-344, 2015.

[4] M. Naehrig, K. Lauter and V. Vaikuntanathan, "Can homomorphic encryption be practical?", In Proceedings of the $3^{\text {rd }}$ ACM workshop on Cloud computing security workshop, Pp. 113-124, 2011.

[5] K. Min Chung, Y. Kalai and S. Vadhan, "Improved delegation of computation using fully homomorphic encryption", In Advances in Cryptology-CRYPTO 2010, Pp. 483-501, 2010.

[6] A. López-Alt, E. Tromer and V. Vaikuntanathan, "On-the-fly multiparty computation on the cloud via multikey fully homomorphic encryption", In Proceedings of the forty-fourth annual ACM symposium on Theory of computing, Pp. 1219-1234, 2012.

[7] M. Van Dijk and A. Juels, "On the impossibility of cryptography alone for privacy-preserving cloud computing", HotSec, Vol. 10, Pp. 1-8, 2010.

[8] C. Aguilar-Melchor, S. Fau, C. Fontaine, G. Gogniat and R. Sirdey, "Recent advances in homomorphic encryption: A possible future for signal processing in the encrypted domain", Signal Processing Magazine, IEEE, Vol. 30, No. 2, Pp. 108-117, 2013.

[9] A. Chatterjee, M. Kaushal and I. Sengupta, "Accelerating sorting of fully homomorphic encrypted data", In INDOCRYPT, Vol. 8250, Pp. 262-273, 2013.

[10] Henning Perl. hcrypt project. This library is accessible at https://github.com/hcrypt-project/libScarab. 
[11] N. Smart and F. Vercauteren, "Fully homomorphic encryption with relatively small key and ciphertext sizes", In Public Key Cryptography PKC 2010, Pp. 420-443, 2010.

[12] D.E. Knuth, "The Art of Computer Programming", Fundamental Algorithms, Addison Wesley Longman Publishing, Vol.1, 1998.

[13] K.E. Batcher, "Sorting networks and their applications", In Spring Joint Computer Conference, Proceedings, Pp. 307-314, 1968.

[14] C. Gentry, "Fully homomorphic encryption using ideal lattices", In: STOC, Pp. 169-178, 2009.

[15] C. Gentry and S. Halevi, "Implementing Gentry's fully-homomorphic encryption scheme", In: EUROCRYPT, Pp.129-148, 2011.

[16] C. Gentry, S. Halevi and N.P. Smart, "Fully homomorphic encryption with polylog overhead", IACR Cryptology ePrint Archive Report, 2011.

[17] C. Gentry, S. Halevi and N.P. Smart, "Homomorphic evaluation of the AES circuit. IACR Cryptology ePrint Archive Goldwasser, S., Micali, S.Probabilistic encryption \&amp; how to play mental poker keeping secret all partial information. In: Proceedings of the Fourteenth Annual ACM Symposium on Theory of Computing, Pp. 365-377. 